\title{
A clinical presentation of drug-induced cardiotoxicity event in an oncohematology patient: A case report
}

\author{
Ainur Bilmakhanbetova ${ }^{1}$, Meruyert Beisenbay ${ }^{1}$, Daulet Marat $^{1}$, and Gulnur Zhakhina ${ }^{1}$ \\ ${ }^{1}$ National Research Center for Oncology and Transplantology
}

November 24, 2020

\author{
Abstract \\ report \\ Ainur Bilmakhanbetova ${ }^{1}$ \\ Meruyert Beisenbay ${ }^{2}$ \\ Daulet Marat ${ }^{3}$ \\ Gulnur Zhakhina ${ }^{4}$ \\ ${ }^{1}$ Center of Radiation and Functional Diagnostics, \\ National Research Oncology Center, \\ Nur-Sultan, Kazakhstan \\ Email: Ainur-0105@mail.ru \\ ${ }^{2}$ Center of Radiation and Functional Diagnostics, \\ National Research Oncology Center, \\ Nur-Sultan, Kazakhstan \\ Email:5340788@mail.ru \\ ${ }^{3}$ Center Multidisciplinary Therapy, Cardiology, \\ National Research Oncology Center, \\ Nur-Sultan, Kazakhstan \\ Email: Daulet.md@mail.ru \\ ${ }_{4}^{4}$ National Research Oncology Center, \\ Nur-Sultan, Kazakhstan. \\ Email: gulnur.zhakhina@nu.edu.kz
}

This case report deals with a clinical case of a patient who underwent inpatient treatment of the underlying disease acute leukemia. In the selection of treatment for complications, medications of various groups were prescribed. This therapy led to the clinical death of the patient, caused by drug-induced QT/QTc prolongation.

Title: A clinical presentation of drug-induced cardiotoxicity event in an oncohematology patient: A case 


\title{
Correspondence
}

Gulnur Zhakhina

National Research Oncology Center

Mailing address: Nur-Sultan, Kazakhstan,

Kerey-Zhanibek khandar street, 3

Email:gulnur.zhakhina@nu.edu.kz

\begin{abstract}
Cardiotoxicity is a term that includes various undesirable cardiovascular events during chemotherapy in hematological cancer patients. Cardiotoxicity can occur both during chemotherapy and at different times after its completion. Its manifestations are diverse, it can proceed without symptoms and be registered only with instrumental and laboratory research, or be accompanied by a severe, sometimes life-threatening clinical picture. In clinical practice doctors, oncohematologists often face the manifestation of cardiac arrhythmias and its conduction disorders in patients. Acute drug toxicity may also develop resulting in drug-induced cardiac arrhythmias. This case report deals with a clinical case of a patient who underwent inpatient treatment of the underlying disease acute leukemia. During the treatment, multiple infectious complications arose. In the selection of treatment for these complications, medications of various groups were prescribed according to vital indications. This therapy, in turn, unfortunately, led to the clinical death of the patient. The cause could be drug-induced QT/QTc prolongation. A young man, who had no history of structural heart disease, became acutely ill, and during the treatment of the underlying disease (polychemotherapy) received side effects from drug groups that led to the patient's clinical death.
\end{abstract}

\section{KEYWORDS}

cardiotoxicity, chemotherapy, QT/QTc prolongation, drug-induced disease

\section{Key Clinical Message}

With the help of a Holter monitor, we diagnosed life-threatening heart rhythm disturbance, which led to the clinical death of the patient. Treatment with different groups of drugs often leads to prolongation of the QTc interval. Early diagnosis will allow us to avoid all these complications and patient death.

\section{Abbreviations}

CBC, complete blood count; CHF, chronic heart failure; ECG, electrocardiogram; LQTS, long QT syndrome; NROC, National Research Oncology Center; PCT, polychemotherapy; SCD, sudden cardiac death; TdP, Torsade de Pointes.

\section{Introduction}

The use of a multicomponent polychemotherapy program in the treatment often leads to the development of cardiotoxicity, which in turn forces to interrupt a more effective treatment regimen and return to it only after treatment of cardiovascular pathology, and sometimes refuse to continue antitumor therapy. Currently, in clinical cardiology, a serious medical problem is the long QT syndrome, which is a risk factor for cardiac arrhythmias and sudden death. Prolongation of the QT interval often manifests itself in episodes of loss of consciousness and often ends in ventricular fibrillation, which is the immediate cause of sudden death (1).

In clinical practice, congenital and acquired forms of long QT are distinguished (2). This paper presents a description of a clinical case of acquired long QT syndrome, which is caused by the use of an unfavorable combination of drugs. The most common drugs that can prolong the QT interval include antiarrhythmic, class IA III, antibacterial (macrolide and fluoroquinolone groups), several antidepressants, psychotropic, and sedatives, antihistamines, diuretics, and lipid-lowering drugs (3). 
The state of the cardiovascular system largely determines the survival rate of patients with oncohematological diseases in critical conditions. Changes in the cardiovascular system in hematological patients in a specialized hospital are detected based on the results of electro-functional research methods (4). In our practice, we have presented a clinical case of a hematological oncological patient with long QT syndrome of non-hereditary, drug-induced genesis. Its identification and description are of great interest in the clinical practice of physicians from the etiology of serious cardiac arrhythmias and conduction disturbances in patients with no history of organic heart disease.

\section{Case description}

Patient K. 1996, male, height $174 \mathrm{~cm}$, weight $62 \mathrm{~kg}$, was in inpatient treatment in the oncohematological department. The disease debuted in October 2016, with weakness, dizziness, the febrile temperature in the evenings, shortness of breath, and swollen cervical lymph nodes. Under the conditions of the NROC, in the department of hematology, the patient was diagnosed with acute lymphoblastic leukemia (option B III) based on:

1. The result of a complete blood count $(\mathrm{CBC})$ : leukocytes -2.4 thousand/ $\mu$, thrombocytopenia -30 thousand $/ \mu \mathrm{l}$, hemoglobin $-65 \mathrm{~g} / \mathrm{l}$, lymphocytes $-50 \%$, monocytes $-1 \%$, neutrophils $-2.9 \%$, blasts $42 \%$.

2. Myelogram: blasts $-80.4 \%$, myelocytes $-0.4 \%$, segmented $-1.6 \%$. All neutrophils $-2 \%$, monocytes $-0.2 \%$, lymphocytes $-14.8 \%$. Plasma cells $-0.2 \%$. The puncture of the bone marrow is moderately cellular. Blast cells of medium to large size with rounded and oval nuclei, with a delicate chromatin structure with 1-2-3 nucleoli. Granulocyte and erythroid sprouts are reduced. No megakaryocytes were found in the preparations.

3. Immunophenotyping of bone marrow. In the examined bone marrow sample, a population of cells CD19 + / CD10 + / CD34- / HLADR- / cytCD22 + / TDTcyt + / cytlgM + Scd22dim + / CD20dim + / CD13- / CD117- / CD33- / sIgM- / CD7- / MPO- / CD3-cyt- , which is typical for acute lymphoblastic leukemia, variant B III is most likely.

4. Molecular-cytogenetic examination (FISH-diagnostics). Bone marrow puncture is a low cell. 150 interphase nuclei with each DNA probe were analyzed. The t (9:22) (q34: q11) MLL / 11q23 translocation was not detected in the analyzed interphase nuclei. He received polychemotherapy (PCT) according to the ALL-2013 KZ protocol.

According to the protocol, the examination of the heart was compulsorily taken on a standard ECG, which revealed frequent ventricular extrasystole, an episode of sustained ventricular tachycardia with a ventricular rate of 166 beats per minute. The patient was consulted by a cardiologist, after which Holter ECG monitoring was prescribed. During the study, in the evening, in the department, the patient lost consciousness and was transferred to oncological intensive care. The patient's condition worsened, clinical death occurred due to cardiac arrhythmias, namely, ventricular pirouette-type tachycardia (Torsade de Pointes), which turned into ventricular fibrillation (Figure 1). Cardiac resuscitation was performed immediately, and the rhythm was restored by Pulsed Electromagnetic Field therapy.

ECG monitoring was carried out in stationary conditions with a duration of $22: 23 \mathrm{~h}$. Sinus rhythm, sinus arrhythmia, average heart rate -77 beats per minute, minimum heart rate -56 beats per minute (22:58), maximum heart rate in sinus rhythm - 144 beats per minute (20:19). 3872 ventricular extrasystoles were registered, of which: 2242 single, bi-tri-quadrigeminal cycles, which in percentage was $2 \%$ ventricular contractions. Life-threatening heart rhythm disturbance was recorded by 1630 episodes of persistent ventricular tachycardia "Torsade de Pointes" (TdP), 1 episode of ventricular fibrillation with a rate of 348 beats per minute lasting 9 minutes, from 20:09 to 20:18 (clinical death) (Figures 1 and 2). The rhythm was restored by the defibrillator. Also found in the amount of 360 single supraventricular extrasystoles. At an average heart rate, the QT interval was $500 \mathrm{msec}$ and the corrected QTc was $560 \mathrm{msec}$. It is known that a prolongation of the corrected QTc interval is a predictor of sudden cardiac death. The maximum R-R - 1.352 sec (22:58), ischemic changes in the ST segment, and the T-wave was not found. 
During polychemotherapy, the patient had multiple infectious complications against the background of myelotoxic agranulocytosis: on the 8th day of hospitalization - febrile neutropenia, gram-positive sepsis (Staphylococcus aureus), and on the 10th day, probable invasive pulmonary aspergillosis. Given the complications, groups of drugs were prescribed for treatment: broad-spectrum antibiotics, antimycotic, hormonal drugs, cytostatics, diuretics, antihistamines, and anticoagulants. All of these drugs have side effects that could lead to a prolongation of the QT interval. Prolongation of the QTc interval during pharmacotherapy in the patient manifested itself as acute cardiotoxicity: at an average heart rate, QTc was $500 \mathrm{msec}$ and a corrected QTc of $560 \mathrm{msec}$ (Figure 3). The patient received antiarrhythmic therapy according to the Cordarone $600 \mathrm{mg} / \mathrm{s}$ scheme for 2 weeks, then $400 \mathrm{mg} / \mathrm{s}$ for 2 weeks, then $200 \mathrm{mg} / \mathrm{s}$ for 3 months.

In dynamics, a control Holter ECG monitoring was prescribed a month later. The study was conducted against the background of antiarrhythmic therapy. Daily ECG monitoring was carried out in stationary conditions with a duration of $23: 20 \mathrm{~h}$. The main rhythm is sinus, sinus arrhythmia, with an average heart rate of 85 beats per minute, a minimum heart rate of 56 beats per minute (03:31), a maximum heart rate of 131 beats per minute (19:40). The ventricular and supraventricular activity was not registered. Tachycardia $-16 \%$, maximum RR interval -1.26 seconds (04:01). QT with the average heart rate is $400 \mathrm{msec}$ and QTc 480 msec. According to the results of Holter ECG monitoring against the background of antiarrhythmic therapy, episodes of rhythm disturbances were not observed.

\section{Discussion}

The list of drugs that are used to treat oncohematological patients is long, since the long road to treatment, the prescription of multicomponent therapy leads to multiple complications, which in turn require correction and treatment, as well as the appointment of additional drugs. Paroxysms of ventricular tachycardia can clinically manifest as episodes of loss of consciousness and often end in ventricular fibrillation, which is the immediate cause of sudden death (5). Thus, when prescribing medications, it is necessary to bear in mind the possibility of increasing the risk of death in patients with an increased likelihood of developing "Torsade de Pointes" and to monitor the duration of the QT interval (6). Prolongation of this interval is often associated with cardiotoxicity and is drug-induced (7).

One of the most important and significant tasks of cardiology is the early detection and treatment of patients with a high risk of sudden cardiac death (SCD). One of the most dangerous diseases with a risk of developing SCD of arrhythmogenic genesis is long QT syndrome (LQTS), in which the risk of SCD reaches $71 \%$ (6). According to a prospective study by the International LQTS Registry, $57 \%$ of SCD cases occur before the age of 20 (7). In 2016, under the auspices of the European Society of Cardiology Committee for the Development of Practice Guidelines, a document was released that talks about the treatment of cancer patients with chemoradiation therapy, as a result of which cardiovascular toxicity occurs (8). The document provides evidence of the severe effects of chemoradiation therapy on the myocardium: myocardial dysfunction and chronic heart failure (CHF), coronary artery disease, heart valve damage, arrhythmias (especially dangerous, induced by drugs that prolong the QT interval), arterial hypertension, thromboembolism, peripheral vascular disease and strokes, pulmonary hypertension, pericarditis. A fairly large group of chemotherapeutic drugs has a cardiotoxic effect, which can be expressed as asymptomatic ECG changes and myocardial infarction, as well as the development of toxic cardiomyopathy with symptoms of severe heart failure $(7,8)$.

The importance of this clinical case lies in the fact that the direction of treatment and the use of drugs may not always have a positive effect on cardiac activity. Side effects and drug incompatibilities can lead to prolongation of the QT/QTc interval. As you know, prolongation of the QT/QTc interval often leads to fatal cardiac arrhythmias and is a predictor of sudden cardiac death. To date, there are a lot of studies that carefully describe the various causes of acquired lengthening of the interval $(5,9,10)$. The QT interval corrected according to the Bazett formula, with a duration of more than $450 \mathrm{~ms}$ in men and more than 470 $\mathrm{ms}$ in women is considered to be elongated, normal - less than 430 and 450, borderline - from 430 to 450 and from 450 to 470 (7). A QT interval greater than $500 \mathrm{~ms}$ is a predictor of ventricular arrhythmias and sudden cardiac death; therefore, it is recommended to immediately discontinue the drugs causing these changes (6). In this clinical case, according to Holter's results, a life-threatening heart rhythm disturbance was revealed, 
namely, an acquired QT/QTc interval lengthening. Cardiac arrhythmias are often detected in patients with cancer who receive chemotherapy (9). They range from rare cardiac arrhythmias to life-threatening arrhythmias or even sudden cardiac death (10). Arrhythmias can lead to trauma in the event of loss of consciousness, heart failure, or cardioembolic stroke in atrial fibrillation, requiring significant adjustments to the patient's treatment regimen (5). Arrhythmia in patients with oncohematological pathology does not have any distinctive features but can be directly associated with a malignant neoplasm, induced by a chemotherapeutic drug, or occur against the background of an existing disease of the cardiovascular system $(5,6)$. This work examines the features of the manifestation of the syndrome, taking into account the patient's diagnosis and treatment tactics of the underlying disease and its complications. Long QTc syndrome is a common side effect of non-cardiac drugs $(5,6)$. To prevent this kind of complications, physicians must consider the potential risks associated with the effect of LP on the QT interval. Patients who receive combinations of drugs that affect the duration of the QT interval should be warned about the need to promptly inform the attending physician about any symptoms that may be manifestations of "Torsade de Pointes". To detect asymptomatic prolongation of the QT interval of more than $500 \mathrm{~ms}$, it is necessary to regularly conduct an electrocardiographic examination. It is important to share cases of cardiac arrhythmias associated with a combination of different drugs to reduce mortality from arrhythmias in a cohort of hematological cancer patients. Given this clinical case, it should be noted that more careful monitoring of the QT/QTc interval during the patient's treatment can have a beneficial effect on the successful outcome of the prescribed therapy (8).

\section{Conclusion}

In the present clinical case, a drug-induced prolongation of the QT interval was identified, which led to the clinical death of the patient. When prescribing a drug that can increase the duration of the QT interval, patients should be warned about the need to promptly inform the attending physician about any symptoms that they may have. Possible manifestations of "Torsade de Pointes" are fainting, paroxysmal heart rhythm disturbances, especially recently developed, palpitations and pre-syncope, pre-syncope without palpitations. To identify the risk of developing heart failure, a regular electrocardiographic examination is necessary. The latent lengthening of the QT interval is greater than 500ms. Thus, when prescribing drugs, it is necessary to remember the possibility of increasing the risk of death of patients. Besides, it is necessary to take into account the increase in the likelihood of developing TdP and monitor the duration of the QT interval. Hematologists must be fully aware of possible cardiac arrhythmias, and close collaboration between cardiologists and hematologists will lead to better cardiovascular risk stratification, monitoring, and treatment.

\section{Authors' Contributions}

A.B. performed a full analysis of data from Holter monitor, monitored the patient's health, wrote this case report; D.M. prescribed Holter monitoring based on ECG result, monitored the patient's health; M.B. performed an echocardiogram of the heart's movement, monitored the patient's health; G.Z. wrote this case report.

\section{Conflict of interest}

Authors declare that they have no conflict of interest.

\section{Ethical approval}

Case reports are often considered not research and do not need IRB approval.

\section{Acknowledgments}

None. No funding to declare.

\section{Reference list}


1. Lee, K. W., Okin, P. M., Kligfield, P., Stein, K. M., \& Lerman, B. B. (1997). Precordial QT dispersion and inducible ventricular tachycardia. American heart journal, 134 (6), 1005-1013.

2. Priori, S. G., Napolitano, C., Diehl, L., \& Schwartz, P. J. (1994). Dispersion of the QT interval. A marker of therapeutic efficacy in the idiopathic long QT syndrome. Circulation, 89 (4), 1681-1689.

3. Nousiainen, T., Jantunen, E., Vanninen, E., Remes, J., Puustinen, J., Rantala, A., . . . Hartikainen, J. (1998). Acute neurohumoral and cardiovascular effects of idarubicin in leukemia patients.European journal of hematology, 61 (5), 347-353.

4. Roden, D. M. (2008). Long-QT syndrome. New England Journal of Medicine, 358 (2), 169-176.

5. Zipes, D. P., Camm, A. J., Borggrefe, M., Buxton, A. E., Chaitman, B., Fromer, M., . . Quinones, M. A. (2006). ACC/AHA/ESC 2006 guidelines for management of patients with ventricular arrhythmias and the prevention of sudden cardiac death - executive summary: a report of the American College of Cardiology/American Heart Association Task Force and the European Society of Cardiology Committee for Practice Guidelines (Writing Committee to Develop Guidelines for Management of Patients With Ventricular Arrhythmias and the Prevention of Sudden Cardiac Death). Journal of the American College of Cardiology, 48 (5), 1064-1108.

6. Day, C. P., McComb, J. M., \& Campbell, R. W. (1990). QT dispersion: an indication of arrhythmia risk in patients with long QT intervals.Heart, 63 (6), 342-344.

7. Bovelli, D., Plataniotis, G., Roila, F., \& Group, E. G. W. (2010). Cardiotoxicity of chemotherapeutic agents and radiotherapy-related heart disease: ESMO Clinical Practice Guidelines. Annals of oncology, 21 (suppl_5), v277-v282.

8. Zamorano, J. L., Lancellotti, P., Rodriguez Muñoz, D., Aboyans, V., Asteggiano, R., Galderisi, M., . . Lyon, A. R. (2016). 2016 ESC Position Paper on cancer treatments and cardiovascular toxicity developed under the auspices of the ESC Committee for Practice Guidelines: The Task Force for cancer treatments and cardiovascular toxicity of the European Society of Cardiology (ESC). European heart journal, 37 (36), 2768-2801.

9. Strevel, E. L., Ing, D. J., \& Siu, L. L. (2007). Molecularly targeted oncology therapeutics and prolongation of the QT interval.Journal of Clinical Oncology, 25 (22), 3362-3371.

10. Shuykova, K. V., Storozhakov, G. I., Gendlin, G. E., \& al., e. (2010). The case of acute anthracyclines cardiotoxicity. In. Abstracts of XXXIII World Congress of the ISH, Jerusalem, Israel.

\section{Appendix}

Figure 1. Ventricular fibrillation with a heart rate of 330 beats per minute. Clinical death of the patient.

Figure 2. Ventricular fibrillation.

Figure 3. Prolongation of the QT / QTc interval of 500/560 msec. 
07-anp

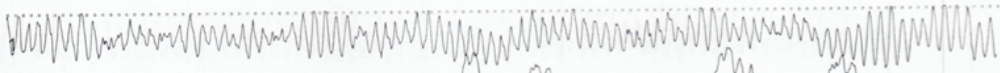

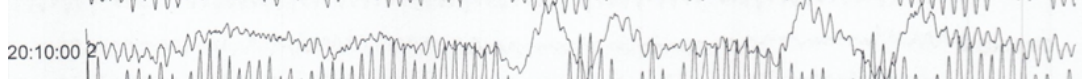

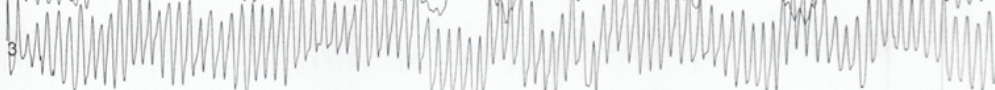

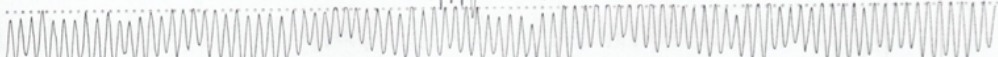

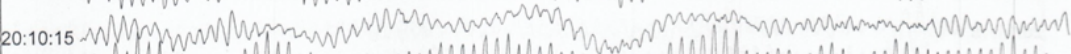

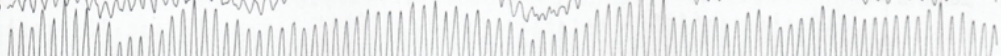

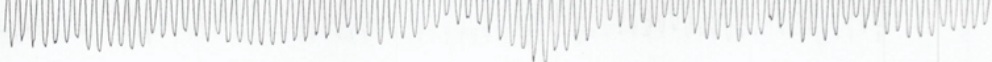

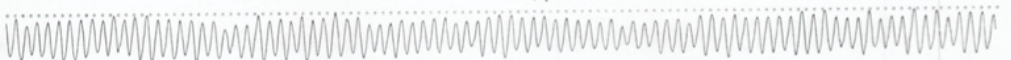

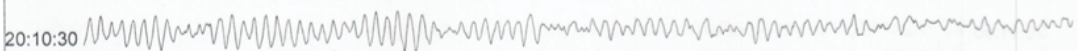

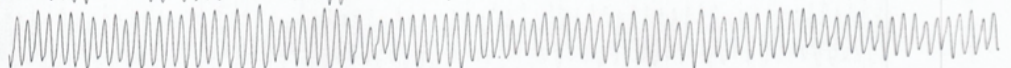

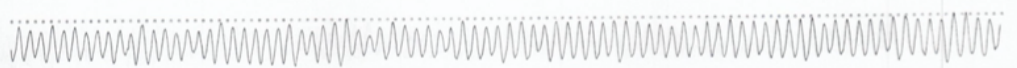

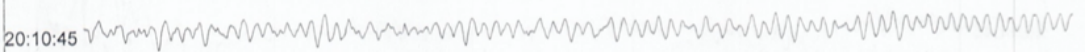

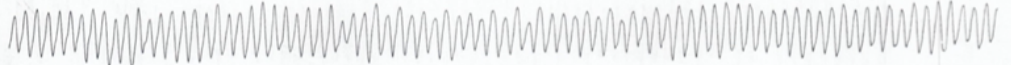
шумьпров 1:41 qhee 330 yg fous

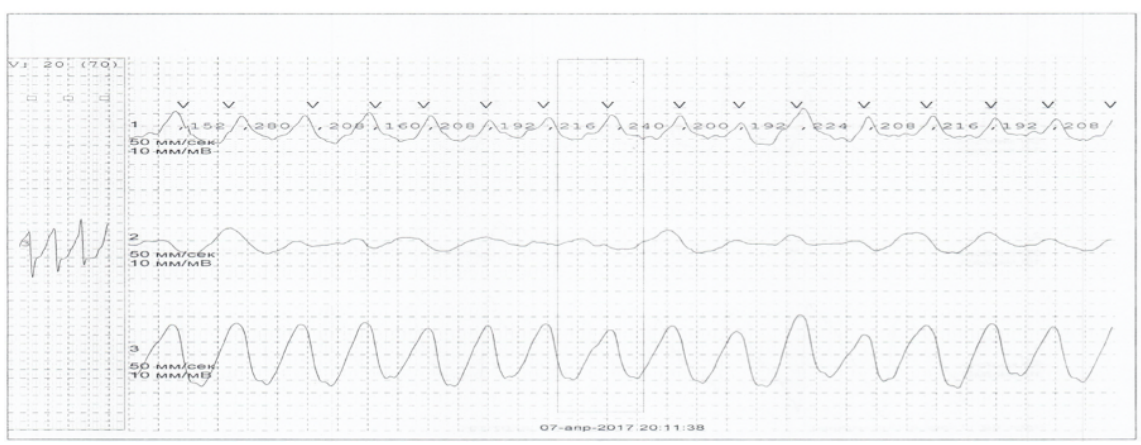




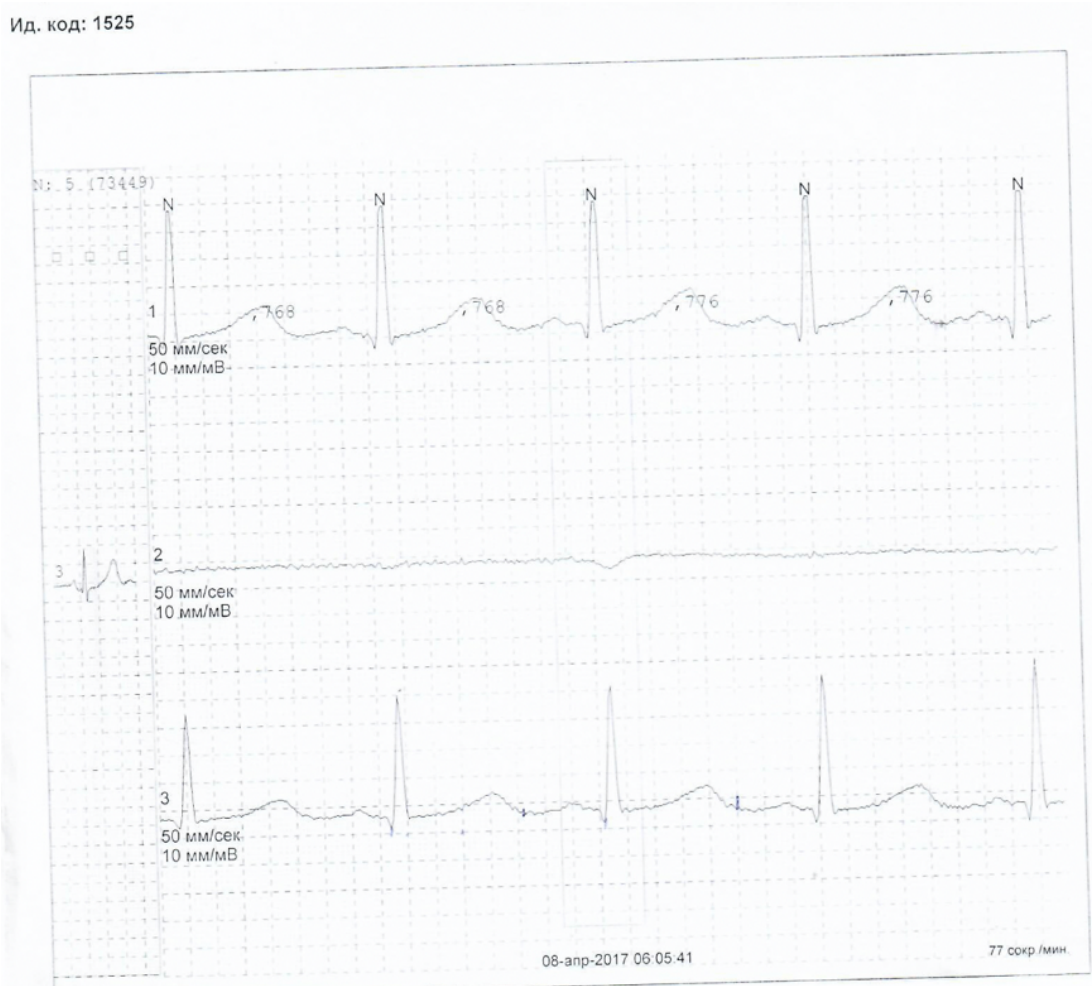

